\title{
The Riemann surfaces of a function and its fractional integral.
}

By William fabian.

1. Introduction. For a many-valued function $f(z)$ of the complex variable $z$, a Riemann surface can be constructed such that, at any point $z$ on the surface, the function has only one value; a function normally multiform, is therefore uniform on a certain Riemann surface.

The operator $D^{-\lambda}$ represents a $\lambda^{\text {th }}$ integral of a function and is defined by ${ }^{1}$

$$
D^{-\lambda}\left(l_{a}\right) f(z)=\frac{1}{\Gamma(\lambda+\gamma)}\left(\frac{d}{d z}\right)^{\gamma} \int_{a}^{z}(z-t)^{\lambda+\gamma-1} f(t) d t,
$$

where $l$ is a simple curve in the plane of the complex variable, along which the integration is carried out. $\lambda$ may be real or complex, and $\gamma$ is the least integer greater than or equal to zero such that $R(\lambda)+\gamma>0, R(\lambda)$ being the real part of $\lambda$.

In this note we are concerned with relations between the Riemann surfaces of a function and its fractional integral.

2. Transformation of Riemann surfaces.

Theorem 1. Let $f(z)$ be analytic within a circle with centre at $a$, and which contains $l$ in its interior. Then a is a branch-point of $D^{-\lambda}\left(l_{a}\right) f(z)$ for non-integral values of $\lambda$.

If $\lambda$ is a rational fraction $r_{/ s}^{\prime}$ expressed in its lowest terms, then $a$ is the vertex of a cycle of $s$ roots.

If $\lambda$ is irrational or complex, then $a$ is the vertex of an infinite number of roots.

Proof. The Taylor series for $f(z)$ at $a$ within the given circle is

$$
f(z)=\sum_{n=0}^{\infty} \frac{f^{(n)}(a)}{n !}(z-a)^{n}
$$

Then applying the operator $D^{-\lambda}$ to each term of this series, we easily find that, within the given circle,

$$
D^{-\lambda}\left(l_{a}\right) f(z)=\sum_{n=0}^{\infty} \frac{f^{(n)}(a)}{\Gamma(\lambda+n+1)}(z-a)^{\lambda+n} .
$$

The conclusion follows immediately.

\footnotetext{
1 Fabian, Thil Mag., 20, 783 (1930).
} 
Theorem 2. Let $f(z)$ be analytic in a bounded region $E$,. except for an isolated singularity within $E$ at a point $p$ different from $a$, at which $f(z)$ can be expanded in a Laurent series.

Then, for non-integral values of $\lambda, p$ is a branch-point of $D^{-\lambda}\left(l_{a}\right) f(z)$, with cycles of an infinite number of roots.

Proof. In the $t$-plane, where $l$ joins the points $t=a$ and $t=z$, let $\mathrm{C}$ be a closed contour through the point $t=z$, which lies wholly in $E$, encloses $p$, and excludes $l$. Denote by $S_{m}$ the curve traced out by a point $t$ which passes along $l$ from $a$ to $z$ and then describes $C m$ times. Then

$$
\begin{aligned}
& D^{-\lambda}\left(S_{m}\right) f(z)=D^{\gamma} D^{-\lambda-\gamma}\left(S_{m}\right) f(z) \\
= & D^{\gamma}\left\{D^{-\lambda-\gamma}\left(l_{a}\right) f(z)+m D^{-\lambda-\gamma}(C) f(z)\right\} \\
= & D^{-\lambda}\left(l_{a}\right) f(z)+m D^{\gamma} D^{-\lambda-\gamma}(C) f(z) \\
= & D^{-\lambda}\left(l_{a}\right) f(z)+m D^{-\lambda-\gamma}(C) f^{(\gamma)}(z),
\end{aligned}
$$

on integrating $D^{-\lambda-\gamma}(C) f(z)$ by parts $\gamma$ times.

By a previous theorem ${ }^{1}$

$$
D^{-\lambda-\gamma}(C) f^{(\gamma)}(z)=2 \pi i \sum_{\sigma=1}^{\infty}(-1)^{\sigma-1} A_{\sigma} \frac{(z-p)^{\lambda-\sigma}}{\Gamma(\lambda-\sigma+1) \cdot(\sigma-1) !},
$$

where $\sum_{\sigma=-\infty}^{\infty} A_{\sigma}(z-p)^{-\sigma}$ is the Laurent series for $f(z)$ at $p$.

The conclusion now follows from (1).

Theorem 3. Let $f(z)$ be analytic in a bounded region $E$ on the Riemann surface associated with $f(z)$, except for a branch-point within $E$ at a point $p$ different from a, at which $f(z)$ can be expanded in a Puiseux series. Let the number of roots of $f(z)$ in the cycle ${ }^{2}$ at $p$ be $r$.

If the Puiseux series for $f(z)$ at $p$ does not contain negative integral powers of $(z-p)$, the number of roots of $D^{-\lambda}\left(l_{a}\right) f(z)$, where $\lambda$ is non. integral, in the corresponding cycle at $p$ does not exceed $r$. If the series contains negative integral powers of $(z-p)$, the number of roots of $D^{-\lambda}\left(l_{a}\right) f(z)$, where $\lambda$ is non-integral, in the corresponding cycle at $p$ is infinite.

1 Fabian : Phil. Mag., 21, 277 (1936).

2 If $f(i)$ has $M$ cycles at $p, f(z)$ is to be regarded as having $M$ branch-points at $p$, and the theorem applies to each of these branch-points separately. 
Proof. On the Riemann surface associated with $f(t)$, let $C$ be a closed contour through the point $t=z$, which lies wholly in $E$, encloses $p$ and excludes $l$, where $l$ joins $a$ and $z$. Denote by $S_{m}$ the curve traced out by a point $t$ which passes along $l$ from $a$ to $z$ and then describes $C m$ times.

As in the proof of Theorem 2, we have

$$
D^{-\lambda}\left(S_{m}\right) f(z)=D^{-\lambda}\left(l_{a}\right) f(z)+m D^{-\lambda-\gamma}(C) f^{(\gamma)}(z) \text {. }
$$

By a previous theorem, ${ }^{1}$ from which the value of $D^{-\lambda-\gamma}(C) f^{(\gamma)}(z)$ can be immediately deduced, it follows that $D^{-\lambda-\gamma}(C) f^{(\gamma)}(z)$, for nonintegral values of $\lambda$, is or is not zero, according as the Puiseux series for $f(z)$ at $p$ does not or does contain negative integral powers of $(z-p)$. The result then follows from (1).

1 Fabian : Phil. Mag., 21, 276 (1936).

14 Grosvenor Avende,

CANONBURY, LONDON, N.5. 